\title{
Effect of Functional Endoscopic Sinus Surgery on Mucociliary Clearance in Patients of Chronic Rhinosinusitis
}

\author{
Shivam Agarwal ${ }^{1}$, Rohit Sharma ${ }^{2}$, Vineet Kumar Sharma ${ }^{3}$, Amit Kumar Rana $^{4} \odot$
}

\begin{abstract}
Introduction: Mucociliary clearance is a nonspecific defense mechanism that removes mucus and other materials from the nose and lungs. During functional endoscopic sinus surgery (FESS) sometimes there is damage to a lot of healthy sinonasal mucosa. This study was carried out to measure the outcome of mucociliary clearance test before and after FESS.

Materials and methods: Study group included 44 patients diagnosed with chronic rhinosinusitis (CRS) posted for FESS. Mucociliary clearance testing was performed with saccharine pellets.

Observations: The age of patients ranged between 10 and 80 years with $25 \%$ between 20 and 30 years and $20 \%$ between 30 and 40 years of age. Forty percent of patients had bilateral nasal obstruction as the primary nasal complaint. Nasal discharge was seen in $30 \%$ of patients. Onethird of patients had complete loss of smell. It was observed that the mean time taken for the concentration of saccharine appreciation in the oropharynx for mucociliary clearance preoperative was 58 minutes. Postoperatively at 6 weeks, it was 14 minutes and 12 minutes at 10 weeks. Discussion and conclusion: Mucociliary clearance as one of the parameters of nasal physiology was evaluated in the patients using saccharine test. Our study revealed after comparing the results in the postoperative period, the percentage was raised from $70 \%$ improvement at the 6 th week to $85 \%$ at the 10 th postoperative week. In our study, we saw that the saccharine test was easy to perform, saccharine was easily available, and it was cost-effective and could be applied to all patients of CRS to assess nasal physiology.
\end{abstract}

Keywords: Cilia, Chronic rhinosinusitis, Mucociliary clearance, Nasal physiology, Nose, Saccharine.

Otorhinolaryngology Clinics: An International Journal (2021): 10.5005/jp-journals-10003-1381

\section{INTRODUCTION}

Nose is the primary sensory organ responsible for olfaction and breathing. It also performs a very important function of mucociliary clearance. Mucociliary clearance is a nonspecific defense mechanism that removes mucus and other materials from the nose and lungs by ciliary and secretary activity of tracheobronchial tree. ${ }^{1}$ Ventilation and drainage are the two essential intrinsic functions of paranasal sinuses. They also warm and moisten the inspired air and also protect against high pressure changes in the nasal region; they lighten the skull weight and also act as resonating chambers. ${ }^{2}$

Chronic rhinosinusitis (CRS) is a chronic inflammatory disease of sinonasal mucosa. It causes significant physical symptoms, has a negative impact on the quality of life, and can substantially impair daily functioning. CRS is a public health problem, affecting approximately $2-5 \%$ of general population. ${ }^{3}$ Alteration in mucociliary clearance leads to nasal blockage and rhinorrhea leads to impaired ciliary activity causing rhinosinusitis with or without nasal polyposis. ${ }^{4}$ Although the condition is not life-threatening, it is costly in terms of expenditure over the medical and surgical management. The major symptom complex of CRS includes nasal obstruction, nasal congestion, facial pressure sensation, rhinorrhea, and hyposmia. The minor symptoms include halitosis, headache, toothache, fatigue, and fever. Nasal endoscopy and computed tomography are the main tools for diagnosing and classifying the severity of CRS. ${ }^{5}$

Functional endoscopic sinus surgery (FESS) is a globally accepted surgical management of CRS if medical management is not effective. ${ }^{6,7}$ During FESS, there is removal and sometimes even damage to a lot of healthy sinonasal mucosa; therefore, the effect of
${ }^{1}$ Department of ENT, Lala Lajpat Rai Memorial Medical College, Meerut, Uttar Pradesh, India

${ }^{2-4}$ Department of Otolaryngology and Head Neck Surgery, SRMS Institute of Medical Sciences, Bareilly, Uttar Pradesh, India

Corresponding Author: Rohit Sharma, Department of Otolaryngology and Head and Neck Surgery, SRMS Institute of Medical Sciences, Bareilly, Uttar Pradesh, India, Phone: +91 9997449995, e-mail: rohitsharma.dr@ gmail.com

How to cite this article: Agarwal S, Sharma R, Sharma VK, et al. Effect of Functional Endoscopic Sinus Surgery on Mucociliary Clearance in Patients of Chronic Rhinosinusitis. Int J Otorhinolaryngol Clin 2021;13(2):37-39.

Source of support: Nil

Conflict of interest: None

FESS on mucociliary clearance is not clear and is a matter of debate. This study was carried out to measure the outcome of mucociliary clearance test before and after FESS in patients of CRS.

\section{Materials and Methods}

This study was carried out in the department of Otolaryngology and Head Neck Surgery in a tertiary care center of Bareilly, UP, India from December 2013 to August 2015. The study was approved by the institutional research and ethics committee. The study group included all the patients diagnosed with CRS with or without polyposis posted for FESS. All the patients underwent detailed history and complete ENT examination. Diagnostic nasal endoscopy and noncontrast enhanced computed tomography scan (NCCT) nose and peripheral nervous system (PNS) were performed in all

() The Author(s). 2021 Open Access This article is distributed under the terms of the Creative Commons Attribution 4.0 International License (https://creativecommons. org/licenses/by-nc/4.0/), which permits unrestricted use, distribution, and non-commercial reproduction in any medium, provided you give appropriate credit to the original author(s) and the source, provide a link to the Creative Commons license, and indicate if changes were made. The Creative Commons Public Domain Dedication waiver (http://creativecommons.org/publicdomain/zero/1.0/) applies to the data made available in this article, unless otherwise stated. 
the cases. Mucociliary clearance testing was performed after the patient was admitted to the ward.

Before performing the test, it was confirmed that the patient has not been exposed to any substance interfering with the test results. A sodium saccharine pellet measuring $2 \mathrm{~mm}$ was placed on the surface of the inferior nasal concha. The patient was told to remain seated with their head straight while breathing normally, without sneezing or nose blowing. The time taken by the patient to appreciate a particular taste in the nasopharynx or oropharynx was noted. FESS was performed in all these patients. All patients were given broad-spectrum antibiotic, nasal decongestant, saline drops, and topical steroid spray for 6 weeks. Cleaning and nasal douching were done with saline after removal of nasal packing and at postoperative follow-up visits. Diagnostic nasal endoscopy and saccharine tests were repeated at 6 weeks postoperative, and findings were recorded and tabulated.

\section{Observations and Results}

Study was performed on 44 patients diagnosed with CRS. The age of patients ranged between 10 and 80 years with $25 \%$ between 20 and 30 years and $20 \%$ between 30 and 40 years of age. The youngest patient was 12 years and oldest was 74 years. Fifty-six percent of patients were males and 44\% were females (Tables 1 and 2).

Table 3 shows that around $40 \%$ had bilateral nasal obstruction as the primary nasal complaint. Nasal discharge was seen in $30 \%$ of patients. One-third of patients had complete loss of smell.

A NCCT nose and PNS was performed in all patients. Sinusitis was present in all the patients and nasal polyposis was seen in only $9 \%$ of patients (Table 4). Table 5 shows the comparison of mucociliary clearance by saccharine test, preoperative and postoperative at 6 and 10 weeks.

Table 1: Age distribution

\begin{tabular}{lc}
\hline Age in years & No. of patients (\%) \\
\hline $10-20$ & $9(20.5)$ \\
$20-30$ & $11(25)$ \\
$30-40$ & $9(20.5)$ \\
$40-50$ & $5(11.4)$ \\
$50-60$ & $7(15.9)$ \\
$>60$ & $3(6.8)$ \\
Total & $44(100)$ \\
\hline
\end{tabular}

Table 2: Sex distribution

\begin{tabular}{lc}
\hline Sex & No. of patients (\%) \\
\hline Male & $25(56.8)$ \\
Female & $19(43.2)$ \\
Total & $44(100)$ \\
\hline
\end{tabular}

Table 3: Nasal obstruction in study groups

\begin{tabular}{lc}
\hline Side & No. of patients (\%) \\
\hline Right & $13(29.5)$ \\
Left & $8(18.2)$ \\
Both & $18(40.9)$ \\
None & $5(11.4)$ \\
Total & $44(100)$ \\
\hline
\end{tabular}

Table 4: Nasal discharge in study groups

\begin{tabular}{lc}
\hline Side & No. of patients (\%) \\
\hline Right & $12(27.3)$ \\
Left & $7(15.9)$ \\
Both & $13(29.5)$ \\
None & $12(27.3)$ \\
Total & $44(100)$ \\
\hline
\end{tabular}

Table 5: Comparison of mucociliary clearance by saccharine test preand postoperatively

\begin{tabular}{lccccc}
\hline & & $\begin{array}{c}\text { Mean } \\
\text { time to }\end{array}$ & & & \\
& Nasal & & & & \\
appreciate & & & Standard \\
sweet taste & Standard & Mean & \\
Time & cavities & in minutes & Deviation & Error & Pvalue \\
\hline Pre-operatively & & 58.35 & 43.75 & 5.5 & \\
6 week post op & & 14.2 & 8.2 & 1.05 & 0.0001 \\
10 week post op & 62 & 12.05 & 5.5 & 0.70 & 0.0001 \\
\hline
\end{tabular}

It was observed that the mean time taken for the concentration of saccharine appreciation in the oropharynx for mucociliary clearance preoperative was 58 minutes. Postoperatively at 6 weeks, it was 14 minutes and 12 minutes at 10 weeks.

\section{Discussion}

The most essential intrinsic functions of nose and paranasal sinus are ventilation, drainage, and maintaining conductive environment of the nose. ${ }^{8,9}$ Mucociliary clearance acts as a proper defense mechanism in maintaining the viability of the human upper respiratory tract and its impairment leads to chronic infections of the nose and PNS.

In our study, there were 44 patients ranging from 10 to 75 years, in which approximately $45 \%$ of the patients were between 20 and 40 years of age. This made us assume that the younger population is more susceptible to CRS. We compared this finding to a study done in the European population where it is observed that $48 \%$ of patients belonged in this age-group. ${ }^{10}$ It was observed that $56 \%$ of patients were males. Chen et al. ${ }^{11}$ in their study on Canadian population stated that prevalence of this disease was slightly higher in females, especially those living in the eastern regions of the world. Another study done by Shashy et al. on a population of Minnesota stated that prevalence of CRS was more in females as compared to males in ratio of $68 \%$ in females to $32 \%$ in males. ${ }^{12}$ A study on Spanish population using saccharine over 249 patients and healthy control group revealed that saccharine test is an easy, inexpensive, and reproducible test for quick assessment of naso-mucociliary clearance. ${ }^{13}$

In our study, we saw that nasal obstruction and discharge were the most common symptoms in the majority of the patients. In another study of Bhattacharya, ${ }^{14}$ it was observed that $54 \%$ of patients who suffered from CRS exhibited nasal obstruction and polyposis as a major problem on their visit to clinician. Ling and Kountakis showed that along with nasal obstruction $84 \%$, postnasal drip $80 \%$, and facial congestion $79 \%$ were also the major presenting symptoms. ${ }^{15}$ In our study, $40 \%$ of patients had both nasal obstruction and nasal discharge as a primary symptom causing impairment of nasal mucociliary clearance. This was 
compared to a study which showed that nasal obstruction and nasal discharge were present in $47.3 \%$ of the patients. ${ }^{16}$ Another study done by Min et al. showed that the mean mucociliary test time was 60 minutes. ${ }^{17}$

Mucociliary clearance as one of the parameters of nasal physiology was evaluated in the patients using saccharine test. Among 44 patients suffering from CRS with or without nasal polyposis included in this study, 62 nasal cavities underwent mucociliary clearance assessment and it was seen that a majority of the patients appreciated a sweet taste in the oropharynx in a time period of around 90 minutes. This is compared to a study by Birdi et al. ${ }^{18}$ who analyzed 43 patients who were divided into unilateral and bilateral CRS. They found that the mean time for mucociliary clearance was 55 minutes in both the groups. The normal mucociliary clearance time in a study was 6.99. They stated that a significant change in mucociliary clearance occurred in chronic sinusitis. It was enhanced in the early stages and was significantly slowed down in the late stages. In obstructive disease, it is markedly slowed down but significant improvement occurred after medical and surgical treatment. They went on to say that the saccharine test was an economical, simple, and sensitive test for evaluation of mucociliary clearance which could be used as diagnostic as well as prognostic tool in chronic sinusitis. Shivkumar and Sambauda showed the effectiveness of FESS in patients of CRS with or without nasal polyposis in terms of mucociliary clearance preor postoperatively. They concluded an overall improvement of around $86.6 \%$ in nasal physiology and in relieving from symptoms of CRS. In our study, we found the effectiveness and comprehensiveness of FESS that resulted in significant improvement in mucociliary clearance of all patients of CRS from $45 \%$ preoperatively to $85 \%$ postoperatively after 3 months. ${ }^{19}$

Another study was conducted by Rudmik and Smith depicting the status of mucociliary clearance pre- and postoperatively after FESS. They employed saccharine test and concluded that around $65-80 \%$ of patients suffering from CRS showed characteristic improvement only after FESS irrespective of undergoing long-term medical treatment. ${ }^{20}$ Our study revealed after comparing the results in the postoperative period, the percentage was raised from $70 \%$ improvement at the 6 th week to $85 \%$ at the 10 th postoperative week. In our study, we saw that the saccharine test was easy to perform, saccharine was easily available, and it was cost-effective and could be applied to all patients of CRS to assess nasal physiology.

\section{Conclusion}

Chronic rhinosinusitis is a chronic inflammatory disease of the nose and the paranasal sinus mucosa causing significant physical symptoms. It has a negative impact on the quality of life and can substantially impair the daily functioning of an individual. It is one of the main causes of derangement of nasal physiology hampering olfaction and mucociliary clearance pathway. The saccharine test was helpful in assessing the changes in nasal physiology in form of mucociliary clearance. The test is easy to perform and saccharine is easily available. The test can be performed in an outpatient department as well as bedside to assess nasal physiology parameters. FESS proves as a useful surgery in changing the outcome of mucociliary clearance in patients suffering from CRS.

\section{ORCID}

Amit KRana @ https://orcid.org/0000-0001-8196-5346

Rohit Sharma 가 https://orcid.org/0000-0003-4534-1055 Vinit K Sharma @ https://orcid.org/0000-0001-7843-718X

\section{References}

1. Deborah S, Pratibha KM. Measurement of nasal mucociliary clearance. Clin Res Pulmo. 2014;2(2):1019. Available at: https:// www.jscimedcentral.com/Pulmonology/pulmonology-2-1019.pdf (accessed October 12, 2021).

2. Ogle OE, Weinstock RJ, Friedman E. Surgical anatomy of nasal cavity and paranasal sinuses. Oral Maxillifac Surg Clin N Am 2010;24(2):156166. DOI: 10.1016/j.coms.2012.01.011.

3. Sieron HL, Sommer F, Hoffmann TK et al. Function and physiology of the maxillary sinus. HNO. 2020;68(8):566-572. doi: 10.1007/s00106020-00869-2.

4. Slater A, Smallman LA, Logan AC, Drake-Lee AB. Mucociliary clearance in patients with nasal polyposis. Clin Otolaryngol Allied Sci 1996;21(4):343-47.

5. Alobid I, Bernel-Sprekelsen M, Mulloj J. Chronic rhinosinusitis and nasal polyposis: the role of generic and specific questionnaires on assessing its impact on patient's quality of life. Allergy 2008;63(10):1267-1279. DOI: 10.1111/j.1398-9995.2008.01828.x.

6. Mullol J, Alobid I. Combined oral and intranasal corticosteroid therapy: an advance in the management of nasal polyposis? Ann International Med 2011;154(5):365-367. DOI: 10.7326/0003-4819-154-5-201103010-00011.

7. Guielmany JM, Alobid I, Mullol J. Controversies in the treatment of chronic rhinosinusitis. Expert Rev Respir Med 2010:4(4):447-463. DOI: 10.1586/ers.10.49.

8. Sahim Yilmaz A, Naclerio RM. Anatomy and physiology of upper airway. Proc Am Thrac Soc 2011;8(1):31-39. DOI: 10.1513/pats.201007-050RN.

9. Patel RM, Pinto JM. Olfaction: anatomy, physiology and disease. Clin Anat 2014;27(1):54-60. DOI: 10.1002/ca.22338.

10. Hasten D, Fokkens WJ, Bachert $C$, et al. Chronic rhinosinusitis in Europe- an underestimated disease. A GA ${ }^{2}$ LEN study. Allergy 2011;66(9):1216-1223. DOI: 10.1111/j.1398-9995.2011.02646.x.

11. Chen $Y$, Dales R, Lin M. The epidemiology of chronic rhinosinusitis in Canadians. Laryngoscope 2003;113(7):1199-1205. DOI: 10.1097/00005537-200307000-00016.

12. Shashy RG, Moore EJ, Weaver A. Prevalence of the chronic sinusitis diagnosis in Olmsted County, Minnesota. Arch Otolaryngol Head Neck Surg 2004;130(3):320-323. DOI: 10.1001/archotol.130.3.320.

13. Valía PL, Valero FC, Pardo JM, Rentero DB, Monte CG. Test de la sacarina en el estudio del aclaramiento mucociliar. Valores de referencia en una población española [Saccharin test for the study of mucociliary clearance: Reference values for a Spanish population]. Arch Bronconeumol. 2008;44(10):540-5. PMID: 19006634.

14. Bhattacharya N. Symptoms and disease severity differences between nasal septum deviation and Chronic rhinosinusitis. Otolaryngol Head Neck Surg 2005:133(2):173-177. DOI: 10.1016/ j.otohns.2005.03.082.

15. Ling $\mathrm{FT}$, Kountakis SE. Important clinical symptoms in patients undergoing endoscopic sinus surgery for chronic rhinosinusitis. Laryngoscope 2007;117(6):1090-1093. DOI: 10.1097/ MLG.0b013e31804b1a90.

16. Phillips KM, Hoehle LP, Bergmark RW, et al. Association between nasal obstruction and risk of depression in chronic rhinosinusitis. Otolaryngol Head Neck Surg 2017;157(1):150-155. DOI: $10.1177 / 0194599817696294$.

17. Min YG, Yun YS, Song BH, et al. Recovery of nasal physiology after functional endoscopic sinus surgery: olfaction and mucociliary transport. ORL J Otorhinolaryngol Relat Spec 1995;57(5):264-268. DOI: 10.1159/000276755.

18. Birdi SM, Singh S, Singh A. Mucociliary clearance in chronic sinusitis. Indian J Otolaryngol Head Neck Surg 1998;50(1):15-19. DOI: 10.1007/ BF02996761.

19. ShivakumarT, Sambauda AP. Retrospective analysis of the effectiveness of functional endoscopic sinus surgery in treatment of adult chronic rhinosinusitis refractory to medical treatment. Indian J Otolaryngol Head Neck Surg 2011;63(4):321-324. DOI: 10.1007/s12070-011-0238-2.

20. Rudmik L, Smith TL. Olfactory improvement after endoscopic sinus surgery. Curr Opin Otolaryngol Head Neck Surg 2012;20(1):29-32. DOI: 10.1097/MOO.0b013e32834dfb3d. 